\title{
Drug Utilization Profile of Antibacterial Drugs for the Treatment of Hospitalized Patients in a Teaching and a Non-Teaching Hospital of Ujjain District
}

\author{
Sandeep Singh Bhadoriya ${ }^{1 *}$, Rekha Sharma ${ }^{2}$ and Narendra Madoriya ${ }^{3}$ \\ ${ }^{1}$ Vikrant Institute of Pharmacy, Indore, India \\ ${ }^{2}$ NIMS University, Jaipur, India \\ ${ }^{3}$ Vikram University, Ujjain, India
}

\begin{abstract}
Objectives: The Hospital is a setting where a large number of drugs are administered to patients and the costs of hospitalization and drug treatment are high. Information on drug utilization in Hospitals and non-teaching hospitals are lacking in Ujjain. The present study was carried out to obtain information on the, drug utilization during the study period in 100 bed -days.

Methods: This cross-sectional study about antibacterial drugs was carried out in two hospitals (teaching and nonteaching) for duration of six months. Prescription case records of 710 in-patients were included in this study and were subjected to analysis according to WHO indicators.

Result: In $80.8 \%$ of patients antibiotics were used which indicates that gross over use of drugs. The average number of antibiotic prescription was 1.99.Polypharmacy in $81.08 \%$ patients was reported. Brand name prescribing $(80.03 \%)$ is far ahead than generic prescribing. Parentral route $(57.4 \%)$ of administration is preferred over oral route .The most frequently prescribed antibiotics were Amikacin, Ceftriaxone.
\end{abstract}

Keywords: Non-teaching hospitals; Antibiotics; Prescription pattern

\section{Introduction}

Drug utilization study is the marketing, distribution, prescription, and use of drugs in a society, with main focus on the resulting medical, social and economic consequences. Antibiotics are the most frequently prescribed drugs among hospitalized patients and it is a need of time to rationalize its use about antibacterial drugs [1].

\section{Material and Method}

This cross-sectional study about antibacterial drugs was carried out in two hospitals (teaching and non-teaching) for duration of six months [1]. Prescription case records of 710 in-patients were included in this study and were subjected to analysis according to WHO indicators.

\section{Result}

In $80.8 \%$ of patients antibiotics were used which indicates that gross over use of drugs. The average number of antibiotic prescription was 1.99 . Polypharmacy in $81.08 \%$ patients was reported. Brand name prescribing $(80.03 \%)$ is far ahead than generic prescribing. Parentral route $(57.4 \%)$ of administration is preferred over oral route. The most frequently prescribed antibiotics were Amikacin, Ceftriaxone. Most commonly prescribed FDC was Ceftriaxone with Tazobactam. The number of antibiotics matches with Essential drug list of WHO and Govt. of India were 17 and 14 respectively. In only $6.4 \%$ patient's culture and sensitivity report was available.

\section{Discussion and Conclusion}

Prescribing trends indicates marked polypharmacy with overuse of antibiotics. Parentral route was preferred over oral route. Brand name prescribing fairly predominates. There is a need to prescribe antibiotic on the basis of its appropriateness, efficacy, safety and cost of therapy which will also minimize the risk of resistance.

\section{Reference}

1. MR Ain, N Shahzad, M Aqil, MS Alam, R Khanam (2010) Drug utilization pattern of antibacterials used in ear, nose and throat outpatient and inpatient departments of a university hospital at New Delhi. India J Pharm Bioall sci 2 8-12.
${ }^{*}$ Corresponding author: Sandeep Singh Bhadoriya, Vikrant Institute of Pharmacy, Indore, India, E-mail: sandeepbhadoriya10@gmail.com

Received April 10, 2013; Accepted June 12, 2013; Published June 15, 2013

Citation: Bhadoriya SS, Sharma R, Madoriya N (2013) Drug Utilization Profile of Antibacterial Drugs for the Treatment of Hospitalized Patients in a Teaching and a Non-Teaching Hospital of Ujjain District. Clin Exp Pharmacol S4: 007. doi:10.4172/2161-1459.S4-007

Copyright: @ 2013 Bhadoriya SS, et al. This is an open-access article distributed under the terms of the Creative Commons Attribution License, which permits unrestricted use, distribution, and reproduction in any medium, provided the original author and source are credited. 